\title{
A microscopic calculation of neutrino neutral reaction on ${ }^{4} \mathrm{He}$
}

\author{
Doron Gazit* and Nir Barnea* \\ *The Racah Institute of Physics, The Hebrew University, 91904 Jerusalem, Israel.
}

\begin{abstract}
An ab-initio calculation of the inelastic neutral reaction of neutrino on ${ }^{4} \mathrm{He}$ is presented [1], including realistic nuclear force and full final state interaction among the four nucleons. The calculation uses the powerful combintation of the Lorentz integral transform (LIT) method and the hyperspherical-harmonic effective interaction approach (EIHH). The neutrino - nucleus interaction is taken in the impulse approximation. With respect to previous calculations [2], [3], the current work predicts an increased reaction cross-section by $10 \%-30 \%$ for neutrino temperature up to 15 $\mathrm{MeV}$.
\end{abstract}

Neutrino reactions with nuclear targets have an important part in many physical phenomena. In astrophysics, for example, neutrino-nuclei interactions have a main role in supernova explosion and the nucleosynthesis of the elements. The neutral inelastic reactions of ${ }^{4} \mathrm{He}$ with $v_{x}\left(\overline{v_{x}}\right)(x=e, \mu, \tau)$ have a part in these two phenomena. Core collapse supernovae are widely accepted to be a neutrino driven explosion of a massive star. When the iron core of a massive star becomes gravitationally unstable it collapses until short-range nuclear forces halt the collapse and drive an outgoing shock through the outer layers of the core and the inner envelope. However, the shock loses energy through dissociation of iron nuclei and neutrino radiation, and gradually stalls, it becomes an accretion shock. It is believed, but to date not proven, that the shock is then revived as neutrinos emitted from the collapsed core (the proto-neutron star) deposit energy in the matter behind the shock to reverse the flow to an outgoing shock which explodes the star. Hydrodynamic simulations fail in reviving the shock [4], [5]. One of the ways to solve this problem is through enhancing the neutrinos-matter coupling.

The matter behind the shock is composed mainly of protons, neutrons, electrons, and ${ }^{4} \mathrm{He}$ nuclei. In contrast to the fairly known cross-sections of neutrinos with electrons and nucleons, the interaction of neutrinos with ${ }^{4} \mathrm{He}$ is not accurately known. The current work is the first realistic microscopic calculation of this cross-section.

In their way to the stalled shock, the electron-neutrinos remain in equilibrium with matter for a longer period than their heavy-flavor counterparts, due to the larger cross sections for scattering of electrons and because of charge current reactions. Thus the heavy-flavor neutrinos decouple from deeper within the star, where temperatures are higher. Typical calculations yield temperatures of $\sim 10 \mathrm{MeV}$ for $\mu$ - and $\tau$ - neutrinos [6], which is approximately twice the temperature of electron-neutrinos. Consequently, there is a considerable amount of $v_{\mu, \tau}$ with energies above $20 \mathrm{MeV}$ that can dissociate the ${ }^{4} \mathrm{He}$ through neutral reaction.

Theoretical understanding of neutrino-nucleus scattering process is achieved through 
TABLE 1. Flavor and temperature averaged inclusive inelastic cross-section and energy transfer cross-section calculated. The temperatures are given in $\mathrm{MeV}$, the crosssections in $10^{-42} \mathrm{~cm}^{2}$, and the energy transfer cross-sections in $10^{-40} \mathrm{~cm}^{2} \mathrm{MeV}$

\begin{tabular}{|c|c|c|c|}
\hline \multirow[t]{2}{*}{$\mathrm{T}[\mathrm{MeV}]$} & \multicolumn{2}{|c|}{$\langle\sigma\rangle_{T}\left[10^{-42} \mathrm{~cm}^{2}\right]$} & \multirow{2}{*}{$\begin{array}{c}\langle\sigma \omega\rangle_{T} \\
{\left[10^{-40} \mathrm{~cm}^{2} \mathrm{MeV}\right.}\end{array}$} \\
\hline & This work & Ref. [2] & \\
\hline 4 & $2.09(-3)$ & - & $5.27(-4)$ \\
\hline 6 & $3.84(-2)$ & $3.87(-2)$ & $1.03(-2)$ \\
\hline 8 & $2.25(-1)$ & $2.14(-1)$ & $6.30(-2)$ \\
\hline 10 & $7.85(-1)$ & $6.78(-1)$ & $2.30(-1)$ \\
\hline 12 & 2.05 & 1.63 & $6.27(-1)$ \\
\hline 14 & 4.45 & - & 1.42 \\
\hline 16 & 8.52 & - & 2.84 \\
\hline
\end{tabular}

perturbation theory of the weak interaction model. In the limit of small momentum transfer (compared to the $\mathrm{Z}$ particle rest mass), the effective Hamiltonian can be written as a current-current interaction: $\hat{H}_{W}=\frac{G}{\sqrt{2}} \int d^{3} x j_{\mu}(\vec{x}) J^{\mu}(\vec{x})$, where $G$ is the Fermi weak coupling constant, $j_{\mu}(\vec{x})$ is the leptonic current, and $J^{\mu}$ is the hadronic current. The matrix element of the leptonic current is $\left\langle f\left|j_{\mu}\right| i\right\rangle=l_{\mu} e^{-i \vec{q} \cdot \vec{x}}$, where $\vec{q}$ is the momentum transfer and $l_{\mu}=\bar{u}\left(k_{v^{\prime}}\right) \gamma_{\mu}\left(1-\gamma_{5}\right) u\left(k_{v}\right)$.

The nuclear current matrix elements consists of one body weak currents, but also many body corrections due to meson exchange. The many-body currents are a result of meson exchange between the nucleons. The current work is done in the impulse approximation, thus taking into account only one-body terms. In order to estimate this approximation, we refer to studies of inclusive electron scattering off ${ }^{4} \mathrm{He}$ [7], where it is shown that isovector electromagnetic two-body currents, which are proportional to the electroweak vector currents, produce a strong enhancement of the transverse response at low and intermediate energies. In the current calculation, the vector part is almost negligible with respect to the axial part, and the two-body axial currents are expected to give small contributions [8]. The one-body currents connect the ${ }^{4} \mathrm{He}$ ground state and final state wave functions. In order to calculate the cross-section in a percentage level accuracy, one needs a solid estimate of these wave functions.

The differential cross-section is given by Fermi's golden rule, and is proportional to the response functions of the Coulomb, longitudinal, transverse electric and transverse magnetic multipole operators. The response functions are calculated by combining two powerful tools: the Lorentz integral transform (LIT) method [9] and the effective interaction hyperspherical harmonics (EIHH) method [10]. First we use the LIT method in order to convert the scattering problem into a bound state like problem, and then the EIHH method is used to solve the resulting equations. Using this procedure we solve the final state interaction problem avoiding continuum wave functions. The combination of the EIHH and LIT methods brings to a rapid convergence in the response functions when increasing the effective interaction model space.

In Table 1 we present the calculated total temperature averaged cross-section, $\langle\sigma\rangle_{T}=$ 
$\frac{1}{2} \frac{1}{A}\left\langle\sigma_{v}+\sigma_{\bar{v}}\right\rangle_{T}$, and energy transfer cross-section, $\langle\sigma \omega\rangle_{T}=\frac{1}{2} \frac{1}{A}\left\langle\omega \sigma_{v}+\omega \sigma_{\bar{v}}\right\rangle_{T}$, as a function of the neutrinos' temperature. Also presented are earlier results by Woosley et. al. [2]. It can be seen that the current work predicts an enhancement of about $10 \%-30 \%$ in the cross-section.

The energy transfer cross-section was fitted by Haxton to the formula [3],

$$
\langle\sigma \omega\rangle_{T}=\alpha\left(\frac{T-T_{0}}{10 \mathrm{MeV}}\right)^{\beta}
$$

with the parameters $\alpha=0.62 \cdot 10^{-40} \mathrm{~cm}^{2} \mathrm{MeV}, T_{0}=2.54 \mathrm{MeV}, \beta=3.82$. A similar fit to our results yields $\alpha=0.64 \cdot 10^{-40} \mathrm{~cm}^{2} \mathrm{MeV}, T_{0}=2.05 \mathrm{MeV}, \beta=4.46$. It can be seen that the current work predicts a stronger temperature dependence of the cross sections. For example, a $15 \%$ differnce between these calculations at $T=10 \mathrm{Mev}$, grows to a $50 \%$ difference at $T=16 \mathrm{MeV}$.

In conclusion, a detailed realistic calculation of the inelastic neutrino- ${ }^{4} \mathrm{He}$ neutral scattering cross-section is given. The calculation was done in the impulse approximation with numerical accuracy of about $1 \%$. The different approximations used here should result in about $10 \%$ error, mainly due to many-body currents, which were not considered in the current work.

The effect of these results on the supernova explosion mechanism should be checked through hydrodynamic simulations, of various progenitors. Nonetheless, it is clear that our results facilitate a stronger neutrino-matter coupling in the supernova environment. First, our calculations predict an enhanced cross section by $10 \%-30 \%$ with respect to previous estimates. Second, we obtained steeper dependence of the energy transfer cross-section on the neutrino's temperature. Thus, supporting the observation that the core temperature is a critical parameter in the explosion process. It is important to notice that the energy-transfer due to inelastic reactions are $1-2$ orders of magnitude larger than the elastic reactions, ergo the inelastic cross-section are important to an accurate description of the Helium shell temperature.

The Authors would like thank W. Leidemann, G. Orlandini, and L. Marcucci for their help and advice. This work was supported by the ISRAEL SCIENCE FOUNDATION (grant no 202/02).

\section{REFERENCES}

1. Gazit, D., and Barnea, N., Phys. Rev. C (accepted for publication) (2004).

2. Woosley, S. E., Hartmann, D. H., Hoffman, R. D., and Haxton, W. C., Astrophys. J., 356, 272 (1990).

3. Haxton, W. C., Phys. Rev. Lett., 60, 1999 (1988).

4. Liebendorfer, M., Mezzacappa, A., Thielemann, F.-K., Messer, O. B., Hix, W. R., and Bruenn, S., Phys. Rev. D, 63, 103004 (2001).

5. Buras, R., M., M. R., Janka, H.-T., , and Kilfonidis, K., Phys. Rev. Lett., 90, 241101 (2003).

6. Woosley, S. E., Pinto, P. A., and Ensman, L., Astrophys. J., 324, 466 (1988).

7. Carlson, J., Jourdan, J., Schiavilla, R., and Sick, I., Phys. Rev. C, 65, 024002 (2002).

8. Schiavilla, R., Carlson, J., and Paris, M., Phys. Rev. C, 67, 032501 (2003).

9. Efros, V. D., Leidemann, W., and Orlandini, G., Phys. Lett. B, 338, 130 (1994).

10. Barnea, N., Leidemann, W., and Orlandini, G., Phys. Rev. C, 61, 054001 (2000). 